www.jmscr.igmpublication.org

Index Copernicus Value: 79.54

ISSN (e)-2347-176x ISSN (p) 2455-0450

crossrefDOI: https://dx.doi.org/10.18535/jmscr/v7i1.116

\title{
Speciation, Biofilm production and Antibiotic resistance pattern of Coagulase negative Staphylococci isolated from Neonatal septicemia
}

\author{
Authors \\ Dr B. Senthilselvan, DCH, MDVL ${ }^{1}$, Dr B. Subitha $\mathrm{MD}^{2}$ \\ ${ }^{1}$ Senior Assistant Professor, Department of Dermatology, Thoothukudi Medical College, Thoothukudi \\ ${ }^{2}$ Associate Professor, Department of Microbiology, Thanjavur Medical College, Thanjavur \\ Corresponding Author \\ Dr B.Subitha MD \\ Phone no: 9444176479, Email: subithatuticorin@gmail.com
}

\begin{abstract}
Nowadays Coagulase-Negative Staphylococci (CoNS) has become one of the major pathogen for neonatal septicemia as a result of increased use of intravascular devices and other invasive procedures. This prospective study was conducted to study about species distribution, biofilm production and antibiotic resistance pattern of Coagulase-Negative Staphylococci isolated from Neonatal Septicemia. During the ten months study period 68 CoNS were isolated from neonatal septicemia. Speciation of CONS was done by simple, inexpensive biochemical tests and biofilm production was detected by Tube Method and Congo red agar method. On speciation of CoNS Staphylococcus epidermidis (53\%) was the common isolate followed by Staphylococcus haemolyticus (41\%), Staphylococcus warneri (3\%) and Staphylococcus schleiferi (3\%) CoNS isolates showed significant resistance to Ampicillin (82\%), Clindamycin(76\%), Cotrimoxazole (74\%) Erythromycin (74\%), and Methicillin (76\%.). CoNS isolates revealed 100\% sensitivity to Vancomycin. CoNS isolates (53\%) were biofilm producers by Congo Red Agar method and (47\%) were biofilm producers by Tube adherence method. Due to their high antibiotic resistance and increased virulence of CoNS leads to increased morbidity and mortality in neonates. Preventive measures like hand hygiene, aseptic management of intravenous line should be followed to reduce the spread of virulent CoNS.

Keywords: CoNS, Speciation, Biofilm production.
\end{abstract}

\section{Introduction}

Neonatal septicemia remains one of the important causes of mortality and morbidity in neonates even after the introduction of new broad spectrum antimicrobial agents, advanced measures for diagnosis and treatment. ${ }^{(1)}$ Neonatal septicemia is classified into early onset septicemia (EOS) and late onset septicemia (LOS) based on the age at onset. Neonatal sepsis is caused by a variety of
Gram-positive as well as Gram-negative bacteria, and sometimes yeasts. ${ }^{(2)}$ In the last two decades, the isolation of Gram-positive organisms has increased significantly. CoNS are the major cause of sepsis in neonatal intensive care units (NICU) worldwide $^{(3)}$. With the advancement of medical sciences, especially with the increasing use of medical devices, the infections caused by CoNS are rapidly increased. ${ }^{(4)}$ 
$\mathrm{S}$. epidermidis is the most frequently isolated species of CoNS in bacteremia cases, accounting for $50 \%$ to $80 \%$ ofCoNS isolates. Other commonly recovered species include $\mathrm{S}$. haemolyticus, S. saprophytiicus, S. lugdunensis, S. warneri, S. cohinii, S.schleiferi and S. hominis ${ }^{(5,6)}$. CoNS are often resistant to multiple antibiotics. Methicillin resistance among CoNS is particularly important due to resistance to all beta-lactam agents and cross resistance to other anti-microbial classes. ${ }^{(5,6,7)}$ Main virulence determinant for CoNS is biofilm formation. Biofilm consists of multilayered cell clusters embedded in a matrix of extracellular polysaccharide which facilitates the adherence of these microorganisms to biomedical surfaces and protect them from host immune system and antimicrobial therapy ${ }^{(8,9)}$

As neonatal septicemia is a life-threatening emergency and delay in diagnosis and treatment with the appropriate antibiotics may have grave consequences. The characterization of CoNS up to species level, their antimicrobial properties and biofilm production is very important for the treatment of neonatal sepsis. ${ }^{(10,11)}$

\begin{tabular}{|l|c|c|c|c|c|}
\hline Species & $\begin{array}{c}\text { Tube coagulase } \\
\text { test }\end{array}$ & $\begin{array}{c}\text { Ornithine } \\
\text { decarboxylase test }\end{array}$ & Urease test & $\begin{array}{c}\text { Mannose } \\
\text { fermentation test }\end{array}$ & $\begin{array}{c}\text { Novobiocin } \\
\text { sensitivity } 5 \mu \mathrm{g}\end{array}$ \\
\hline S.epidermidis & - & + & + & + & $\mathrm{S}$ \\
\hline S.saprophyticus & - & - & + & - & $\mathrm{R}$ \\
\hline S.haemolyticus & - & - & - & - & $\mathrm{S}$ \\
\hline S. lugdunensis & - & + & + & + & $\mathrm{S}$ \\
\hline S. warneri & - & - & + & - & $\mathrm{S}$ \\
\hline S.cohnii & - & - & + & - & $\mathrm{R}$ \\
\hline S.schleiferi & - & - & - & + & $\mathrm{S}$ \\
\hline
\end{tabular}

Susceptibility to antibiotics (concentration in $\mu \mathrm{g}$ ) Ampicillin $(10 \mu \mathrm{g})$, Liniezolid $(30 \mu \mathrm{g})$, Cotrimoxazole $(25 \mu \mathrm{g}) \quad$ Erythromycin $(15 \mu \mathrm{g})$ Clindamycin $(10 \mu \mathrm{g})$, Cefoxitin $(30 \mu \mathrm{g})$ (Hi Media) were performed on Mueller-Hinton agar plates by Kirby-Bauer disc diffusion method. Zone diameter was measured and interpreted as per the Clinical and Laboratory Standards Institute (CLSI) guidelines.

Phenotypic characterization of biofilm formation by Congo Red Agar Method and Tube Method. ${ }^{(14,}$ 15,16)

\section{Materials and methods}

This prospective study was conducted over the period of 9 months in a Tertiary care hospital. During the study period 68 coagulase negative Staphylococci that fit with the laboratoryconfirmed bloodstream infection criteria defined by the Center for Disease Control and Prevention were isolated from Neonatal septicemia.

Identification and speciation of isolates

The isolates were initially identified by colony morphology, Gram staining, catalase, slide and tube coagulase test and acid formation from mannitol. Speciation of CoNS was done by urease test, mannose fermentation test, Novobiocin sensitivity test, ornithine decarboxylase test. These simple, inexpensive and easy to perform tests were selected from the scheme of Kloos and Schleifer to identify CoNS species. ${ }^{(12,13)}$
Tube method

A loopful of bacterial suspension from overnight culture was inoculated into the $10 \mathrm{ml}$ of Trypticase Soy Broth with $1 \%$ glucose and incubated for $24 \mathrm{~h}$ at $37{ }^{\circ} \mathrm{C}$. The culture tubes were decanted. Then the tubes were washed with phosphate buffer solution ( $\mathrm{pH}$ 7.3). After drying, the tubes were stained with $0.1 \%$ crystal violet. After incubation for $10 \mathrm{~min}$, excess stain was removed and tubes were washed away with water multiple times. Tubes were then kept in inverted position and finally observed for biofilm 
formation. Biofilm formation was considered positive when a visible film lined the wall and bottom of the tube.

Congo red agar method

This test is based on the property of Congo red to stain polysaccharides black. The composition of the medium are BHI (37 gms/L), sucrose (50 gms/L), agar no.1 (10 gms/L) and congo red stain (0.8 gms/L). CoNS isolates were plated oncongo red agar plate and were incubated at $37{ }^{\circ} \mathrm{C}$ for 24 h.. The biofilm forming strains produced black colonies while non-forming strains developed red colonies.

\section{Results}

During the study period $68 \mathrm{CoNS}$ were isolated from neonatal septicemia and they fitted with the laboratory-confirmed bloodstream infection criteria defined by the Center for Disease Control and Prevention (CDC).

In neonatal septicemia Escherichia coli (22\%)was the most common organism followed by Klebsiella pneumoniae (19\%), Coagulase negative Staphylococcus aureus (16\%), Pseudomonas aeruginosa (16\%) Acinetobacterspp (9\%), Enterobacterspp (6\%), Citrobacterspp (3\%) Staphylococcus aureus (3\%) and enterococci sp (3\%).CoNS was the third most common organism isolated in this study.

On speciation of CoNS Staphylococcus epidermidis $(53 \%)$ was the common isolate followed by Staphylococcus haemolyticus (41\%), Staphylococcus warneri (3\%) and Staphylococcuss chleiferi (3\%) CoNS isolates showed significant resistance to Ampicillin (82\%), Clindamycin (76\%), Cotrimoxazole (74\%) and Erythromycin(74\%).CoNS isolates revealed $100 \%$ sensitivity to Vancomycin. 52 (76\%) CoNS isolates showed Methicillin resistance. Out of $68 \mathrm{CoNS}$ isolates $36(53 \%)$ were biofilm producers by Congo Red Agar method and 32(47\%) were biofilm producers by Tube adherence method.

\section{Chart I:Species distribution of CoNS}

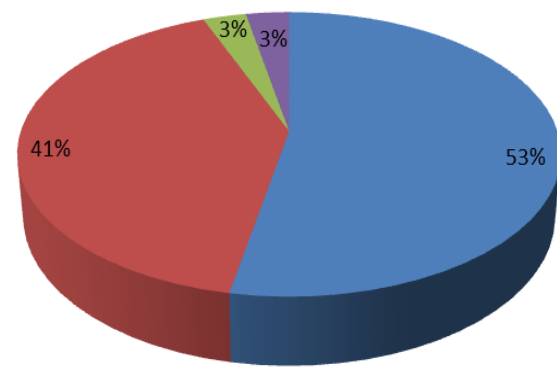

- S.epidermidis

- S.haemolyticus

- S.warneri

S.scheleiferi

\section{Chart II:Antibiotic resistance pattern of CoNS}

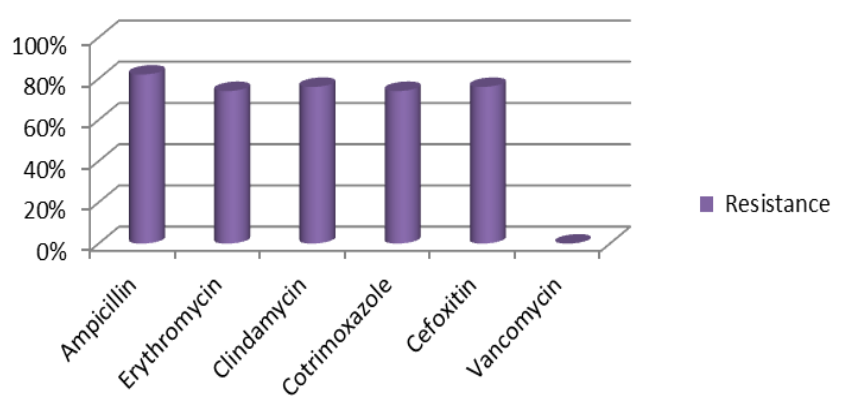

Fig I. Congo Red Agar Method

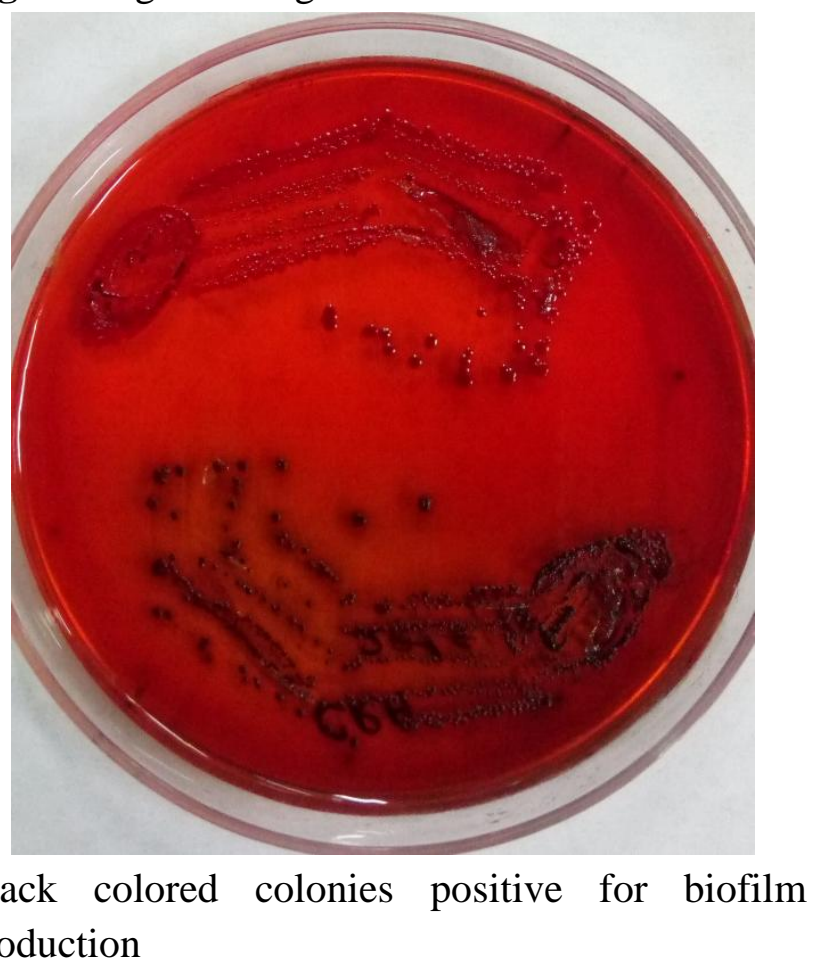




\section{Discussion}

CoNS are normal microbial flora of skin and mucus membrane of human beings. CoNS are considered one of the most common causes of the device related infections in the recent two decades, especially in extreme age group of patients such as neonates and old age.

In the present study CoNS were the third most common isolates in neonatal sepsis and68CoNS were isolated from neonatal septicemia.. This distribution pattern correlates well with the results published by Shahsanam Gheibi et al 2008 who reported that CoNS was the commonest cause of both early (48.8\%) and late onset (69.8\%) neonatal sepsis followed by Klebsiella, and E coli. ${ }^{(17)}$.Marchant et al and Vijaya Shivanna et al (66.6\%), also identified CoNSas the commonest causative organism for neonatal sepsis.

The increasing prevalence of CoNS infections is attributable to their increasing antibiotic resistance and their ability to form biofilms on foreign bodies such as intravascular catheters. ${ }^{(18)}$

In the present study Staphlococcus epidermidis (53\%) was the common isolate followed by Staphlococcus haemolyticus (41\%). The studies conducted in clinically significant CoNS isolates from various samples by Vijayashri et al, Surekha et al, Saroj Golia et al and Manjushree et al reported S.epidermidis as the most frequent isolate (40\%), (,44.8\%) (46.3\%) and (58\%) followed by (S.haemolyticus, (26\%) (19.7\%) (20.1\%), and $(15 \%)$ and S.saprophyticus, (15\%), (27.1\%) (28.4\%), and (21\%)respectively. In the present study S. saprophyticus was not obtained because we studied CoNS isolated only from neonatal sepsis, as S.saprophyticusis frequently isolated from urinary tract .infections.

In our study, antibiotic susceptibility testing showed maximum resistance to ampicillin (82\%), and all CoNS isolate were sensitive to vancomycin. Similarly Surekha et al and Vijayashri et al reported ampicillin resistance $(88.5 \%)(79 \%)$, and no resistance to vancomycin. Manjushree et al also reported maximum resistance to Penicillin (98.08\%; and all the isolates were susceptible to Vancomycin. But Saroj Golia identified ampicillin resistance (88\%) and vancomycin resistance $(1.5 \%)$. In the present study Methicillin resistance was observed in $76 \%$. This was concordant with Vijayashri et al who reported Methicillin resistance in $72 \%$ of CoNS and Surekha et al reported $64.6 \%$ of Methicillin resistance. But lower Methicillin resistance 19.2 $\%$ was published by Manjushree et al.

The ability of biofilm formation seems to play an essential role in the virulence of coagulasenegative staphylococci (CoNS). ${ }^{(21)}$ The most clearly characterized component of staphylococcal biofilms is the polysaccharide intercellular adhesin (PIA) encoded by the icaADBC operon. In the present study $53 \%$ were Biofilm producers by CRA method and $47 \%$ by TM method. Similar findings were observed by Vijayashri et al who reported $(69 \%)$ asbiofilm producers by CRA method and $33 \%$ by TM method. Riyaz Sheriff et al also reported biofilm production in $75 \%$ of CoNS isolates. Riyaz Sheriff et al and T Mathur et al identified that Tissue culture plate method was very sensitive and very specific method to detect the biofilm production But in the present study biofilm production was detected by CRA method and TM method only. The high prevalence of MR-CoNS and biofilm producing strains demonstrated in this study indicates to follow the preventive measures to reduce the spread of virulent CoNS.

\section{Conclusion}

Increase in the use of medical devices, such as intravascular catheters and prosthetic devices, in neonates, will increase the prevalence of CoNS in neonatal septicemia.. The antibiotic resistance and biofilm production of CoNS produce negative impact on clinical outcome of neonatal sepsis. So it is necessary to study about the speciation, antibiotic resistance pattern and virulence determinants of CoNS isolated in neonatal septicemia. 


\section{References}

1. Shivanna, Sunkappa, The rising trend of coagulase-negative staphylococci in neonatal septicemia, Indian Journal of Pathology and Microbiology, Volume59/ issue 4 pages $510-514$

2. J L Yu, S X Wu, H Q Jia, Study on Antimicrobial Susceptibility of Bacteria Causing Neonatal Infections: A 12 Year Study (1987 - 1998) Singapore Med J 2001 Vol 42(3) : 107-110

3. Shreya Singh, Sujeesh Sebastian, Benu Dhawan, The Changing Face of Coagulase-Negative

Staphylococci:Diagnostic And Therapeutic Challenges, Manipal Journal of Medical Sciences | June 2016 | Volume 1, 29-37.

4. Klingenberg C1, Aarag E, Rønnestad A, Sollid JE, Abrahamsen, Coagulasenegative staphylococcal sepsis in neonates. Association between antibiotic resistance, biofilm formation and the host inflammatory response. Pediatr Infect Dis J. 2005 Sep;24(9):817-22.

5. SarojGolia, Deepali Bhimacharya Telsang, Asha S. Kamath B, Speciation of clinically significant coagulase negative staphylococci and their antibiotic resistant patterns in a tertiary care hospital, International Journal of Research in Medical Sciences, 2015 May;3(5):12421246.

6. Surekha. Y. Asangi, Mariraj. J, Sathyanarayan. M.S, Speciation of clinically significant Coagulase Negative Staphylococci and their antibiotic resistant patterns in a tertiary care hospital. Int $\mathbf{J}$ Biol Med Res. 2011; 2(3): 735-739.

7. Mila V.S, Dr.S.Manjusree, Sri. Ashraf Perilacode, Speciation of the Coagulase Negative Staphylococci Isolated from Various Clinical Samples and their Antimicrobial Resistance Pattern at a Tertiary Care Hospital, JMSCR
Vol||05||Issue||03||Page

19063-19068|| March 2017.

8. T Mathur, $\mathrm{S}$ Singhal, $\mathrm{S}$ Khan, DJ Upadhyay, T Fatma, A Rattan, Detection of biofilm formation among the clinical isolates of staphylococci: an evaluation of three different screening methods ,Indian Journal of Medical Microbiology, (2006) 24 (1):25-9,

9. Riyaz Sheriff1, A Sheena2, Assessment of Biofilm Production in Clinically Significant Isolates of Staphylococcus epidermidis and Comparison of Qualitative and Quantitative Methods of Biofilm Production in a Tertiary Care Hospital, International Journal of Scientific Study |September 2016 | Vol 4 | Issue 6 41-46

10. Bizzarro, Shabanova, Neonatal Sepsis 2004-2013: The Rise and Fall of Coagulase-Negative Staphylococci, The Journal of pediatric ,Volume 166,Issue 5, 2015 pages 1193-1199

11. Elizabeth A. Marchant, Guilaine K. Boyce, Manish Sadarangani, and Pascal M. Lavoie,Neonatal Sepsis due to CoagulaseNegative Staphylococci Clinical and Developmental Immunology Volume 2013, 10 pages 586076

12. S. S. Vijayasri Badampudi, Surya Kirani KRL, Rajyalakshmi, Coagulase Negative Staphylococcus; the Speciation and Biofilm Production of Coagulase Negative Staphylococcal Isolates from Clinically Significant Specimens and their Antibiogram. JKIMSU, Vol. 5, No. 2, April-June 2016 69-78.

13. Usha MG1, Shwetha DC, Vishwanath G. Speciation of coagulase negative Staphylococcal isolates from clinically significant specimens and their antibiogram. Indian J Pathol Microbiol. 2013 Jul-Sep;56(3):258-60. doi: 10.4103/0377-4929.120383. 
14. Lok Bahadur Shrestha, Narayan Raj Bhattarai and BasudhaKhanal, Antibiotic resistance and biofilm formation among coagulase-negative staphylococci isolated from clinical samples at a tertiary care hospital of eastern Nepal. Antimicrobial Resistance \& Infection Control2017 86:89

15. Oliveira, De Lourdes Rs Cunha, Comparison of methods for the detection of biofilm production in coagulasenegative staphylococci, Research Gate,2010,Volume/page260

16. Martini, Hörner, Investigation of biofilm formation in coagulase-negative staphylococci isolated from platelet concentrate bags, Rev Inst Med Trop Sao Paulo,2016, Volume 58

17. Shahsanam Gheibi, Mohammad Karamyyar, Most Common Cause of Neonatal Septicemia in Urmia, Iran, Iran J Pediatr Sep 2008; Vol 18 ( No 3), Pp:237243.

18. Astha Agarwal, Amita Jain, Association between drug resistance and production biofilim in Staphylococci, indian J Pathol Microbiol 2013 Jul-Sep:56(3)258-260. 